the addition of $\mathrm{C}$ at concentrations of $3,5,7$ and $9 \mathrm{mmol} / \mathrm{l}$. After 24 hours every hour an optical density of the broth at $580 \mathrm{~nm}$ was measured. The concentration of $C$ was determined in samples before and after cultivation. The concentration of $\mathrm{C}$ was determined in 37 patients, which were included in 3 groups: 1 - "classical" staphylococcal infection (abscess, phlegmon, carbuncle, mastitis, hydradenitis); 2 - secondary infection of wounds with staphylococci; 3 - "not staphylococcal" infections. To determine the level of $\mathrm{C}$ in the culture medium or serum, an enzymatic method was used. Statistical processing of data was carried out using the paired version of Student's t-test.

It was found that $\mathrm{C}$ in all concentrations does not have a bactericidal effect on Staphylococcus spp. Before cultivation of $S$. aureus the level of C was $3.16 \pm 0.06$ and after $-2.69 \pm 0.04 \mathrm{mmol} / 1(\mathrm{p}<0.05)$. Such decrease may be due to the fact that $S$. aureus includes in its metabolism the disrepared diphosphate necessary for the synthesis of the cell wall. Under cultivation of $S$. aureus in the presence of $\mathrm{C}$ the accumulation of biomass was more intense than in a medium without $\mathrm{C}$. A direct relationship between the accumulation of the biomass of the microorganism and the level of $\mathrm{C}$ was shown. In assessing the kinetics of growth of $S$. epidermidis, a similar picture was established. A feature of $S$. epidermidis was an increase in the biomass of cells in a stationary growth phase in the presence of $7 \mathrm{mmol} / \mathrm{l}$ of C.

In patients of the $1^{\text {st }}$ group the level of $\mathrm{C}$ was $4.6 \pm 0.3$ $2^{\text {nd }}-3.28 \pm 0.26 ; 3^{\text {rd }}-4.10 \pm 0.37 \mathrm{mmol} / 1$. In general, the level of $\mathrm{C}$ in patients of the compared groups corresponds to the age norm. However, in patients of the $2^{\text {nd }}$ group concentration of $\mathrm{C}$ significantly differs from the values of the $1^{\text {st }}$ group. We assume that in a secondarily infected wound the processes metabolism of microorganisms proceed more intensively, as a result of which $\mathrm{C}$ can be utilized more by staphylococci, which leads to decrease in its concentration.

Thus, staphylococci are able to include in their metabolism human C, which may be necessary for them for plastic purposes.

\subsection{9}

doi: $10.15789 / 2220-7619-2018-4-9.29$

\section{SENSITIVITY OF BIOFILM CULTURES KLEBSIELLA spp. TO CIPROFLOXACIN}

T.V. Tunik, E.I. Ivanova, E.V. Grigorova, U.M. Nemchenko, Z.I. Budnikova

Scientific Center for Family Health and Human Reproduction Problems, Irkutsk, Russia

In the study researched the effect of 10-, 100-, 1000-fold values of the minimum inhibitory concentration $\left(\mathrm{MIC}_{90}=\right.$ $2 \mu \mathrm{g} / \mathrm{ml}$, literature data), of the antimicrobial preparation ciprofloxacin on Klebsiella spp. autostrains isolated from coprological probes of kids under 5 years old. The experiment included 47 biofilm-forming Klebsiella spp. cultures (28 strains of $K$. pneumoniae and 19 isolates of $K$. oxytoca). A study of the ability of clinical strains to form a biofilm, as well as the influence of a number of concentrations of antibiotic on mature (48-hour) biofilm was carried out in sterile polystyrene plates in a microvolume. Mature biofilm cultures were incubated with ciprofloxacin during the 12 hours under standard conditions with a preliminary purification from plankton cells. The results were considered by optical density of the dye- $1 \%$ crystal-violet bound to the film on a spectrophotometer at a wavelength of $492 \mathrm{~nm}$. The biofilm formation coefficient was calculated as the ratio of the average value of the optical density of the sample to the average value of the optical density of the negative control. The value of the coefficient $\geq 2.1$ was taken as positive.

Biofilms formed by $K$. oxytoca autostems when exposed to ciproflocacin at concentrations exceeding 100 and 1000 -fold the $\mathrm{MIC}_{90}$ were completely destroyed. When exposed of 10 -fold the $\mathrm{MIC}_{90}$, the cells adhering to the surface of the wells formed biofilms that were preserved in $30 \%$ of $K$. oxytoca isolates. Among biofilms formed by strains of $K$. pneumoniae $48.3 \%$ were insensitive to a 10 -fold concentration of ciproflocacin. $35.7 \%$ out of this insensitive isolates were insensitive to a 100 fold concentration of the antibacterial drug. In addition, a strain of K. pneumoniae was detected, which biofilm was not destroyed by a 1000 -fold concentration $(2000 \mu \mathrm{g} / \mathrm{ml})$ of ciprofloxacin. The zone of inhibition of growth of this strain to ciprofloxacin, which investigated by the disco-diffusion method was absent; the strain was characterized as resistant.

Mature biofilms of strains of $K$. pneumoniae were significantly less damaged by exposure to selected concentrations of the antimicrobial drug, ciprofloxacin, compared to $K$. oxytoca isolates.

9.30

doi: $10.15789 / 2220-7619-2018-4-9.30$

THE CORRELATION BETWEEN BIOFILM-FORMATION ABILITY OF KLEBSIELLA spp. AUTOSTRAINS AND ANTIBIOTIC SENSITIVITY OF PLANCTONIC CELLS

T.V. Tunik, E.I. Ivanova, E.V. Grigorova, U.M. Nemchenko, Z.I. Budnikova

Scientific Center for Family Health and Human Reproduction Problems, Irkutsk, Russia

The former study is related to planktonic cells Klebsiella spp. $(\mathrm{n}=117)$ isolated from coprological probes of kids with disbiotic disorder. These cells were isolated using disc-diffusion method to examine the correlation between their sensivity to 11 antibiotics and ability to form firm biofilms in the wells of polystirol microplate.

The study revealed that isolates of $K$. pneumoniae had more autostrains able to form biofilms that $K$. oxytoca $(\mathrm{n}=$ $84,72.6 \%$ and $\mathrm{n}=33,60.6 \%$ respectively). More frequently strains were resistent to amoxicillin (K. oxytoca $-9 \%$, $K$. pneumoniae $-26 \%$ ). The insufficient share of biofilm structures can be explained by vast spread of antimicrobial medication.

All studied autostrains of $K$. oxytoca did not reveal resistence to the majority of antimicrobial medication like imipenem, ertapenem, meropenem, cefepimum, ciprofloxacin, levofloxacin. Strains of $K$. oxytoca which do not form biofilms were completely sensitive to tetracycline, chloramphenicolum, moxifloxacin, doxycycline. In this, intestinal isolates of $K$. oxytoca which form biofilms lowered their sensivity up to $5 \%$ (tetracycline, chloramphenicolum, moxifloxacin) and 10\% (doxycycline).

$K$. pneumoniae strains did not reveal resistance to imipenem, ertapenem. Isolates of $K$. pneumonia which do not form biofilms were completely sensitive to moxifloxacin, chloramphenicolum and meropenem. Biofilm-forming strains had lesser sensitivity up to $8.2 ; 3.6 ; 3.3 ; 1.6 \%$ respectively. Sensitivity of $K$. pneumoniae was $95.7 \%$ to levofloxacin. Sensitivity of $K$. pneumoniae autostrains was 95.7\% and for biofilm-forming strains was lowered up to $10.4 \%$. Sensitivity of non-biofilm isolates of $K$. pneumoniae to doxycycline and ciprofloxacin was $91.3 \%$, and for non-biofilm $-84.4 ; 73.8 \%$ respectively.

The study revealed that planktone cells Klebsiella spp. are able to form biofilm what makes them resistent to most common antibiotics. 